\title{
Separating Inorganics from the Non-metal Fraction of the Processed Waste PCBS Using Heavy Liquid Separation
}

\author{
Amit Kumar $^{1^{\star}}$, Maria Holuszko ${ }^{1}$ and Travis Janke ${ }^{2}$ \\ ${ }^{1}$ NBK Institute of Mining Engineering, University of British Columbia, 517-6350 Stores Road Vancouver, BC, Canada, V6T1Z4 \\ ${ }^{2}$ Ronin8 Technologies Limited, Richmond, BC, Canada
}

"Corresponding author: Amit Kumar, 1NBK Institute of Mining Engineering, University of British Columbia, 517-6350 Stores Road Vancouver, BC, Canada, V6T1Z4, Tel: +16043799093; E-mail: amit.kumar.ism@gmail.com

Received date: August 13, 2018; Accepted date: September 10, 2018; Published date: September 17, 2018

Copyright: (c) 2018 Kumar A, et al. This is an open access article distributed under the terms of the Creative Commons Attribution License, which permits unrestricted use, distribution and reproduction in any medium, provided the original author and source are credited.

\begin{abstract}
Electronic waste is one of the most investigated waste streams around the world due to the presence of valuable metals and hazardous chemicals. Printed circuit boards (PCB) represent a significant amount of this stream which consists of metals such as copper, silver, gold and palladium, and a nonmetal fraction (NMF). The NMF represents approximately $70 \%$ by mass of the total PCB consisting of fiberglass or cellulose as reinforcing materials and epoxy, phenolic or polyester as resins. This paper presents results of the test work on the separation of inorganics and organics from the nonmetal matrix using heavy liquid separation. A liquid medium of varying specific gravity was used to separate different streams from the NMF based on the density variations, and then analyzed for metal content, loss on ignition and calorific value. Results showed that most of the inorganic materials reported to the density range of $1.8-2.4 \mathrm{~g} / \mathrm{cm}^{3}$ with a low calorific value of $<9 \mathrm{GJ} / \mathrm{t}$ and a mass yield of $30-40 \%$ of total NMF. The fraction with density $<1.8 \mathrm{~g} / \mathrm{cm}^{3}$ consisting mostly the organic materials had a high calorific value of $>20 \mathrm{GJ} / \mathrm{t}$ and a mass yield of $60-70 \%$ of the total NMF. It also showed that the heavy metals like copper and iron were concentrated in the heaviest density fraction (>2.4 SG). An optical microscope or scanning electron microscope analysis and mechanical strength tests would be required to further test the quality and respective applications of separated products, which will be considered in the follow up research.
\end{abstract}

Keywords: E-waste; Printed circuit boards; Non-metal fraction; Fiberglass; Cakorific value; Loss on ignition

\section{Introduction}

Electronic waste is one of the fastest growing waste streams in the world. The estimated growth rate of e-waste is reported to be $3-10 \%$ [1-4]. The United Nations University (2017) reported that the total ewaste generated in 2016 was 45 million tones and expected to reach 52 million tons by 2021 . The presence of various valuable metals such as copper, silver, gold, palladium, iron, and aluminum provides an economic incentive for recycling, whereas the hazardous metals such as lead and arsenic and flame retardant bearing chemicals require pretreatment before proper disposal $[5,6]$. The most common types of PCBs are flame retardant 2 (FR-2) and flame retardant 4 (FR-4) which uses cellulose reinforcement with phenolic resins, and fiberglass reinforced with epoxy resin respectively [7]. FR-2 boards are mostly used in low-end electronics such as television and radios, whereas FR-4 is widely used in high-end electronics such as desktops, laptops, and cellphones [7,8]. With an approximate value of $40 \%$ of the total e-waste and $6 \%$ fraction by weight, waste printed circuit boards (PCBs) are one of the significant fractions of the e-waste stream $[9,10]$. Kumar et al. showed that the concentration of copper, silver, gold, and palladium in e-waste is 5 to $250 \%$ higher than average mining operations which provide major economic benefits for recycling [11]. The average metal content in PCBs is approximately $30-35 \%$ and rest of the material is referred to as non-metal [12-14]. The metals in PCBs are extracted for their value and the remaining nonmetal fraction (NMF) containing the resins, including reinforcing materials, and residue metal are often sent to landfills $[15,16]$.
The recovery of metals from the waste printed circuit boards can be achieved by physical or chemical processing. Physical processes such as gravity separation, magnetic separation, electrostatic separation, and flotation had been commonly studied in this area [17-23]. Kaya in 2016 showed that printed circuit board particles of size $<5 \mathrm{~mm}$ can be separated into light nonmetallic and heavy metallic fraction using a zigzag classifier. Ning et al. summarized that jigging can achieve a high metal recovery of up to $97 \%$, whereas air classification can remove $97 \%$ of copper in the sink [22]. Using heavy liquid of density $2.89 \mathrm{~g} / \mathrm{cm}^{3}$, Bizzo et al. separated the NMF from the PCB and thus increased the calorific value to $11.63 \mathrm{GJ} / \mathrm{t}$ in the NMF [12]. Chemical recycling based on pyro, hydro and bio-metallurgy has also been utilized for metal recovery [21-23,24,25]. Recently, Tatariants et al. developed a method using dimethyl formamide and ultrasonic treatment at low temperature $\left(\sim 500^{\circ} \mathrm{C}\right)$ to recover metals from the waste printed circuit boards [26]. A gold recovery of purity level $>94 \%$ with $\sim 12$ times higher yield and a copper nanoparticle of size $5 \mathrm{~nm}$ with a yield of $84 \%$ was reported $[27,28]$. On the other hand, recycling of the NMF to separate its components is not usually practiced due to the lower value of the material; hence, landfilling is most commonly used method of disposal $[15,29]$. However, landfilling requires large wasted land and can lead to groundwater contamination, negative effect of plant growth, leaching of heavy metals and destruction of microbial balance in soil $[7,30,31]$. The NMF in a PCB matrix is approximately $70 \%$ by mass, which consists mainly of fiberglass $(65 \%)$, resins (32\%) and residual metals $[8,23,32]$. Previous studies suggested that the coarser fraction of the NMF has the highest concentration of resins whereas the finer fraction has the highest concentration of fiberglass and 
residual metals [8]. Alternative use of NMF as secondary materials in construction, paints, and adhesive, fillers for epoxy resins to produce composite boards, partial replacement of inorganic aggregates in concrete, use in plastic molds, production as adsorbent for water treatment, replacing wood flour in the production of phenolic moulding compound and producing nonmetallic plate has been suggested $[8,20,22,30,33,34]$. Guo et al. and Ning et al. listed chemical treatments methods such as pyrolysis, gasification, depolymerization and hydrogenolytic degradation as a recycling method to convert the NMF polymers into chemical feedstocks or fuels. Yousef et al. extracted the woven fiberglass at a temperature of $500 \mathrm{C}$ with chemical and ultrasonic treatment and showed that the average strength of the recycled fiberglass decreased by $48 \%$.

Li et al. suggested the possibility of recycling of fiberglass powder from the resin powder using corona electrostatic separator based on the differences in their shape characteristics [35]. The triboelectric separation was used to separate inorganics (fiberglass) from the nonmetal fraction to improve the quality of the product [36]. Approximately $43 \%$ of inorganics were removed using this process and organics content was increased from $49 \%$ to as high as $76 \%$ in certain product streams. Zhang et al. also suggested that the removal of inorganics from the NMF increased the flexural strength, flexural modulus and impact strength of the high density polyethylene composite and thus improved its usability [37]. This paper presents the results obtained from an attempt to separate various components of the NMF such as the resins, fiberglass and residual metals using heavy liquids. Organic liquids of varying densities $\left(1.2\right.$ to $\left.2.4 \mathrm{~g} / \mathrm{cm}^{3}\right)$ were used for this purpose and the separated components were analyzed for metal content, calorific value, thermal stability, and volatile matter. Previous researchers have utilized heavy liquids of higher densities $\left(\sim 2.9-3.1 \mathrm{~g} / \mathrm{cm}^{3}\right)$ to separate the metallic fraction from the nonmetallic fraction with high efficiency [12]. However, the application of heavy liquid to separate various components of NMF in waste PCB has not been studied. Since the density of resins $\left(1.1-1.5 \mathrm{~g} / \mathrm{cm}^{3}\right)$ are lower than the density of pure fiberglass $\left(2.5 \mathrm{~g} / \mathrm{cm}^{3}\right)$, there is a possibility of producing a relatively cleaner organic and cleaner inorganic streams using this process to upgrade the quality of both and thus improving the usability of these products [38-40].

\section{Material and Methods}

\section{Material}

A representative sample of NMF was received from the Ronin8 Technologies Limited pilot plant located in Richmond, British Columbia representing an actual NMF generated at an industrial scale. The plant processed mixture of FR-2 and FR-4 PCBs obtained from computers, printers, and televisions using gravity separation (wet concentration table) to separate metals from non-metals. A representative sample of $25 \mathrm{~kg}$ NMF was collected using cone and quarter method from a $750 \mathrm{~kg}$ bulk bag lot and was oven dried at $600^{\circ} \mathrm{C}$ for 96 hours to remove any residual moisture.

\section{Equipment and test procedures}

Particle size distribution analysis and sample preparation: Approximately 300 grams of sample were obtained from the dried sample for particle size distribution using dry screening method for 20 minutes. The size distribution was plotted, and $80 \%$ and $50 \%$ cumulative passing size were determined.
Float-sink test: Float-sink tests are standard tests to evaluate the feasibility of using a dense medium as a separation technique. Organic liquids with specific gravities (SG) of 1.2, 1.4, 1.6, 1.8. 2.0 and 2.4 were prepared by mixing tetra bromoethane (SG 2.95) and kerosene (SG 0.8 ). These liquids were used for preliminary testing on the following size fractions: $>850 \mu \mathrm{m}, 600-850 \mu \mathrm{m}, 300-600 \mu \mathrm{m}, 150-300 \mu \mathrm{m}$, and 75-150 $\mu \mathrm{m}$. The separated components were analyzed for loss on ignition. Using differences in loss on ignition results from the preliminary tests, bulk float-sink tests were performed on $>500 \mu \mathrm{m}$ and $75-500 \mu \mathrm{m}$ size fraction using liquids of specific gravities of 1.6, 1.8 and 2.4. The separated components were then used for subsequent analysis. The standard procedure for coal wash ability analysis as defined by American Society for Testing and Materials (ASTM) D4371-06 was used. A schematic of the test is shown in Figure 1.

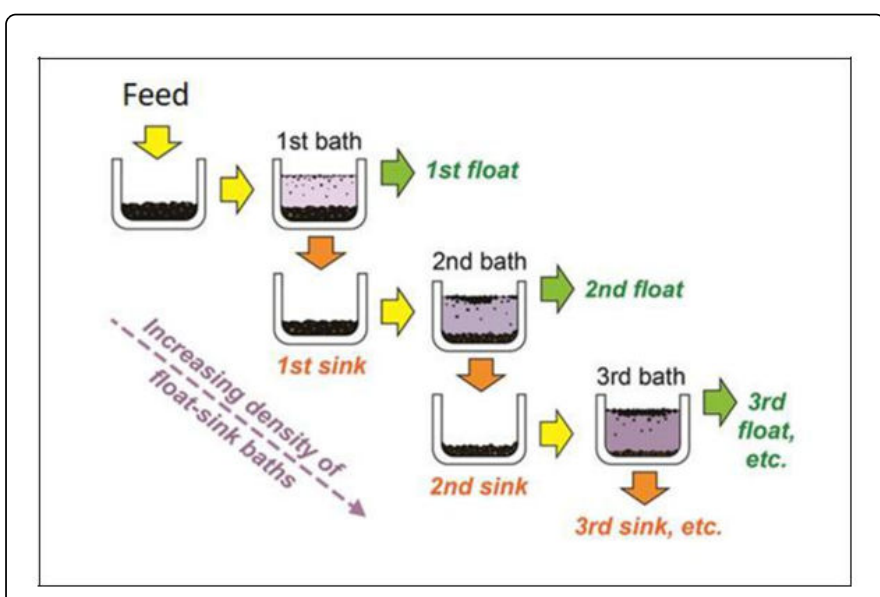

Figure 1: Float and sink test procedure [41].

The sample was placed in the heavy liquid with the lowest specific gravity, mixed and allowed to settle. Floating particles were collected with a scoop lined with fine mesh that allowed excess liquid to drain and the sink material was transferred to the next heavy liquid. Any particles floating at this specific gravity liquid are lighter than the previous liquid but heavier than the current liquid.

The process was repeated for all liquids. All collected fractions were washed with acetone to remove any residual heavy liquid, filtered, dried, weighed and used for subsequent tests.

Loss on ignition (LOI) measurement: Approximately $1 \mathrm{~g}$ sample was heated in the presence of air from room temperature to $500^{\circ} \mathrm{C}$ in 30 was raised from the room temperature $\left(\sim 350^{\circ} \mathrm{C}\right)$ to $9500^{\circ} \mathrm{C}$ at a rate of 100 per minute in the air and nitrogen. Each test was performed using approximately $50 \mathrm{mg}$ samples.

\section{Results and Discussion}

The particle size distribution of the received NMF sample was determined using screening. The distribution is shown in Figure 2. The $80 \%$ passing size (P80) and $50 \%$ passing size (P50) of the sample was estimated to be $819 \mu \mathrm{m}$ and $498 \mu \mathrm{m}$ respectively. 


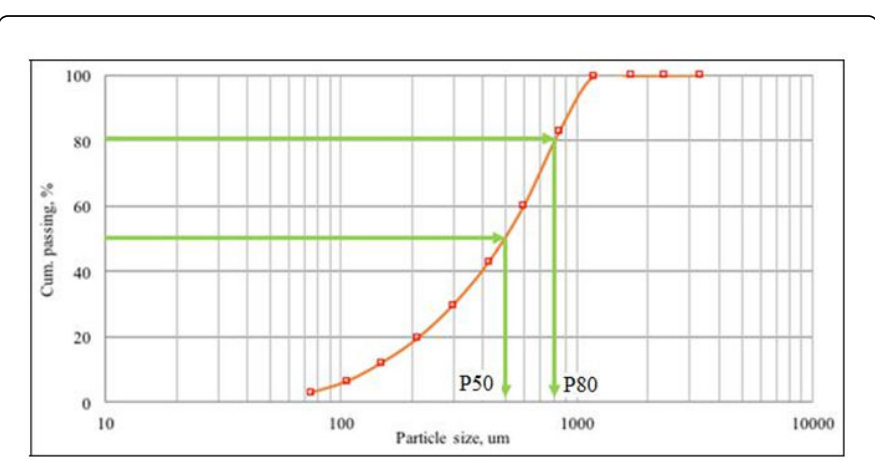

Figure 2: Cumulative weight percent passing distribution vs. particle size for as received NMF.

Preliminary float-sink tests were performed on five size fractions ( $>850 \mu \mathrm{m}, 600-850 \mu \mathrm{m}, 300-600 \mu \mathrm{m}, 150-300 \mu \mathrm{m}$, and 75-150 $\mu \mathrm{m}$ ) to understand the behavior of various size fraction at different specific gravities. The mass loss in these tests ranged from $2-3 \%$ that can be attributed to a) experimental error during test b) dissolution of brominated flame retardants for NMF to the heavy liquids. The concentration of brominated flame retardants needed to be analyzed to confirm this hypothesis which was out of the scope of this study. The mass yields of different density fraction are shown in Figure 3. The lower density fraction should have high resin content due to its low density and the resin content should drop with increasing density. The higher density fraction is expected to have higher fiberglass (inorganics) content. Since the fiberglass is encapsulated in resins and the size reduction process should not be able to produce pure resins fraction, the mass yield should be lower for lowest SG ranges $(<1.2$ and 1.2-1.4) as evident from the graph. It also shows that the yield at highest density is very low as the sample tested was the NMF of the processed waste PCB and most of the metals were already removed. The 1.4-1.6 SG indicates mostly resins fraction with residual fiberglass whereas the 1.8-2.4 SG indicates mostly fiberglass fraction with residual resins. The 1.6-1.8 SG range shows the material with low degree of liberation. The LOI test, discussed later, would be required to show the content of organic resins/inorganic fiberglass in these SG fractions and justify this hypothesis.

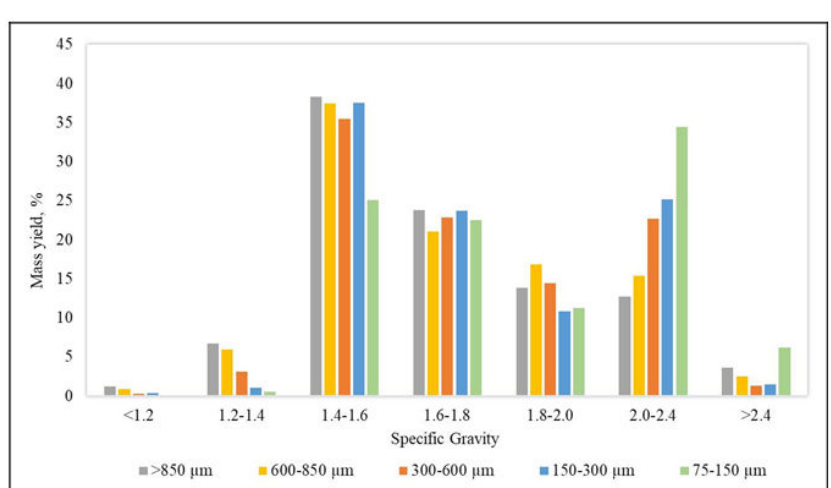

Figure 3: Yield from the preliminary float-sink test at different specific gravities of various size fractions.
Figure 4 shows the loss on ignition (LOI) values for various density categories for each size fraction. The LOI decreased with an increase in the density conforming to the presence of volatile material (resins) in lighter fraction and its decreasing concentration with increasing density. The low LOI of the heaviest density fraction (>2.4 SG) suggests the presence of low organic content.

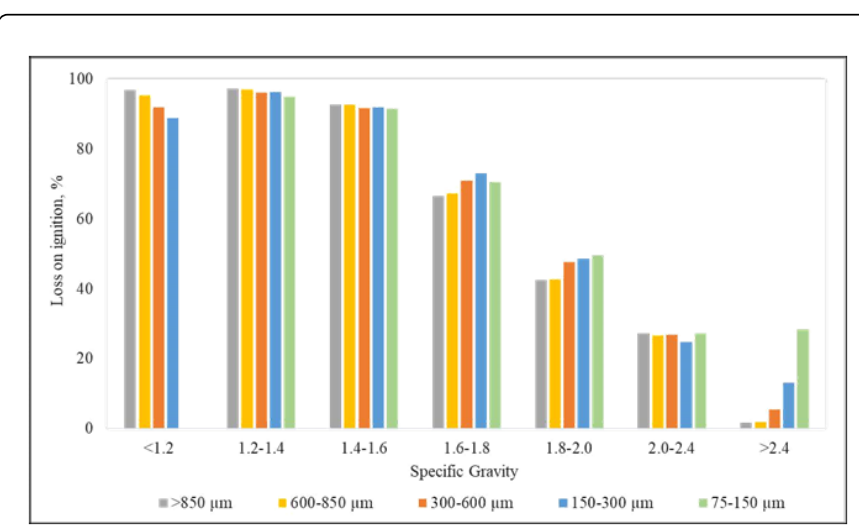

Figure 4: LOI of different specific gravity products for various size fractions.

The increased LOI for the heaviest density fraction (>2.4 SG) for $75-150 \mu \mathrm{m}$ suggests an inefficient separation. Gravity separation is effective for the coarse size fraction and for fine material the settling velocity of particle reduces significantly and thus lowering the separation efficiency $[42,43]$. The similarity in the LOI value for 2.0-2.4 SG and $>2.4$ SG for $75-150 \mu \mathrm{m}$ size fraction suggests that both products were similar in nature and the separation was inefficient for this fraction.

The metal content analysis was also performed to confirm this suggestion which is discussed later in the paper. In most coal preparation plant, coarse particles $(>500 \mu \mathrm{m})$ are treated by gravity separation whereas fine particles $(<500 \mu \mathrm{m})$ are processed using flotation. Gravity separation is used in two or three stages with varying separation densities.

To replicate similar process, the bulk float-sink test and analysis were performed on $>500 \mu \mathrm{m}$ and $75-500 \mu \mathrm{m}$ size fraction with three stage separation. Since the $<75 \mu \mathrm{m}$ sample was too fine and would result in inefficient separation as explained previously, it was not included in the test procedure. The LOI for $<1.2,1.2-1.4$ and 1.4-1.6 SG were similar. Hence the test was performed at 1.6, 1.8 and 2.4 SG. The mass yield and LOI results for the float-sink test on coarse $(>500 \mu \mathrm{m})$ and fine $(75-500 \mu \mathrm{m})$ samples are shown in Figures 5 and 6. The results showed that the mass yield of the $>1.8$ SG range representing mostly the fiberglass was $30-35 \%$ with an LOI of approximately $35 \%$ similar to the results obtained in Figures 3 and 4. 


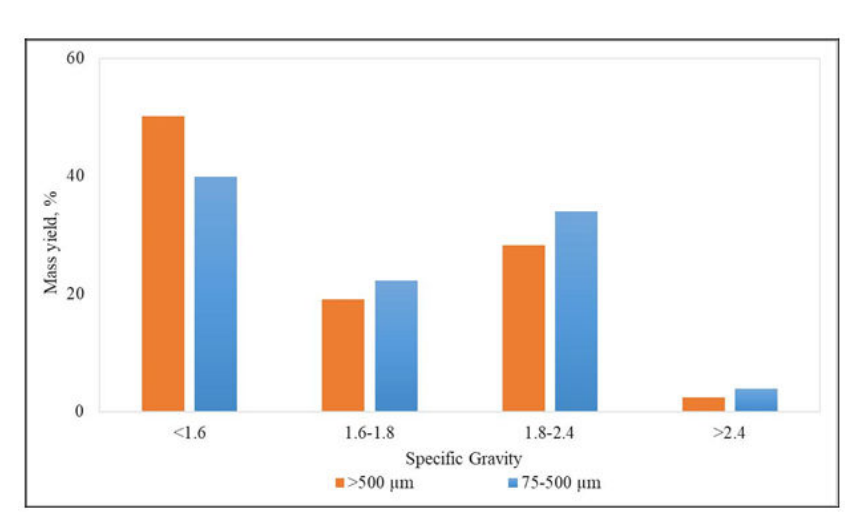

Figure 5: Yield of different specific gravities products for $>500 \mu \mathrm{m}$ and 75-500 $\mu \mathrm{m}$ samples.

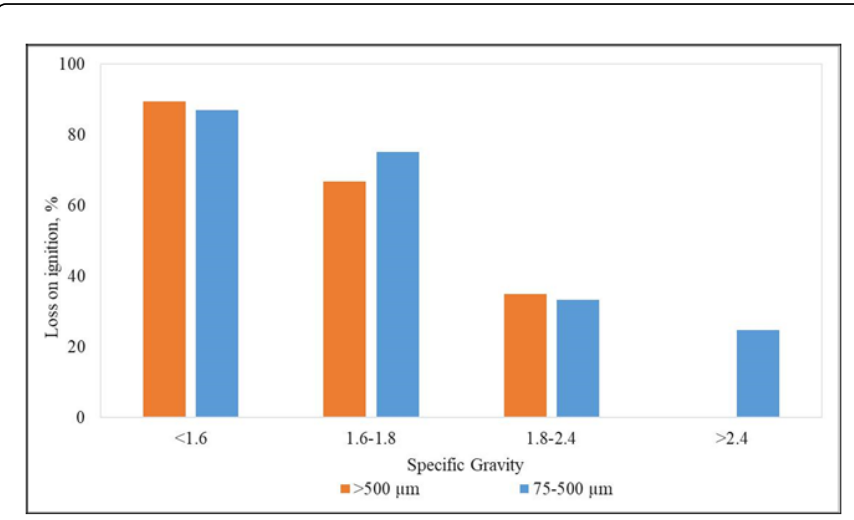

Figure 6: LOI of different specific gravities products for $>500 \mu \mathrm{m}$ and $75-500 \mu \mathrm{m}$ samples.

Marques et al. (2013) reported that the fiberglass and resin content in the NMF was $65 \%$ and $32 \%$ respectively which was somewhat different than the result obtained here. No information was provided regarding the type of PCBs by Marques et al. which influences the material balances [8]. Zheng et al. reported a fiberglass content of $50-70 \%$ with a resin content of $30-50 \%$ for NMF from the processed waste PCB obtained from personal computers, which mostly contains FR-4 type boards which supplements the data obtained by Marques et al. [8,32]. Since, the sample used for this research was a mixed sample (FR-2 and FR-4) most often observed at a typical PCB recycling facility, it would suggest that the fiberglass content of the analyzed sample would be lowed compared to the sample used by Zheng et al. [32].

The LOI data for each density fraction is shown in Figure 6. The LOI values for $<1.6$ and 1.8-2.4 SG fractions are similar, whereas 1.6-1.8 SG and $>2.4$ SG have differences in their LOI values. The 1.6-1.8 SG and $>2.4$ SG fractions for finer samples would have a higher content of misplaced material because of the finer size and lower degree of liberation, thus increasing the overall LOI. These fractions could be treated as the middling fraction and would be re-crushed and reground and should be recirculated back for further separation. Figure 7 shows the recovery of inorganic fiberglass in various density fractions. For the calculation purpose, it was assumed that the LOI residue represented the inorganic fiberglass (with residual metals). However, a complete compositional analysis of the analyzed NMF would be necessary to confirm the exact concentration of fiberglass and various resins. It showed that most of the fiberglass reported to the 1.8-2.4 density fractions as reported from the LOI analysis. The minor recoveries in the lower density indicate insufficient liberation. Similar analysis on the feed materials showed that the fiberglass content for $>500 \mu \mathrm{m}$ and $75-500 \mu \mathrm{m}$ were $32.6 \%$ and $36.3 \%$ respectively.

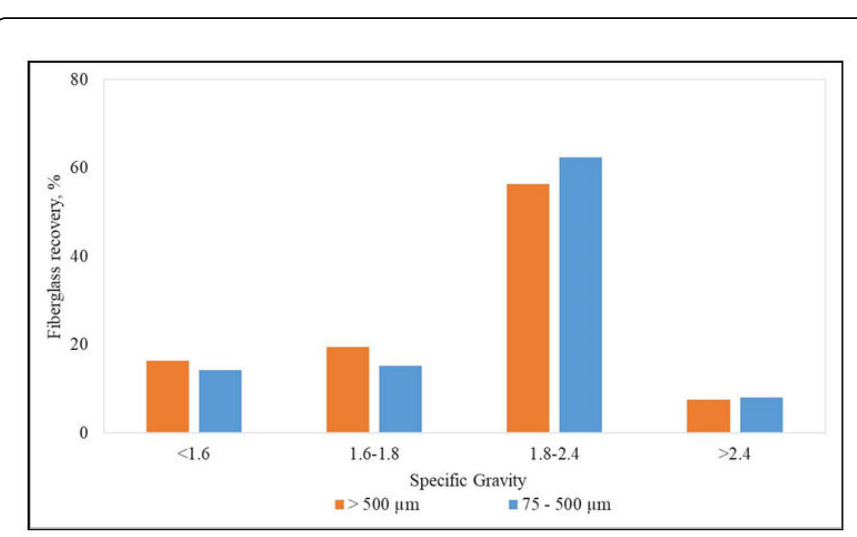

Figure 7: Recovery of inorganic fiberglass in various density fractions for $>500 \mu \mathrm{m}$ and $75-500 \mu \mathrm{m}$ samples.

The $>2.4$ SG fraction for the coarse material had a negative LOI $(-1.42 \%)$ meaning that it has gained mass after igniting to $7500^{\circ} \mathrm{C}$. It suggests that some material (mostly metals) in this fraction was being oxidized (reacting with oxygen) and thus increasing the overall mass and that the total increase in mass due to the material oxidation is greater than the total decrease in mass due to organic volatilization. The negative LOI also suggests the presence of high metal concentration that can be recirculated back to the separation stage for increasing the overall metal recovery. A TGA analysis in the inert atmosphere discussed later was performed to estimate the weight loss in the absence of oxygen.

The calorific value of each density fraction was determined to estimate the energy value of different products. The result for the calorific value is shown in Figure 8. The heaviest fraction ( $>2.4 \mathrm{SG})$ for $>500 \mu \mathrm{m}$ was not tested due to the presence of copper strands. The copper wires in the sample could melt, conduct current and damage the measuring instrumentation. The red line represents the calorific value of the NMF as received $(\sim 15 \mathrm{GJ} / \mathrm{t})$, whereas the green line represents the calorific value of the shredded waste PCB prior to any separation $(\sim 9.5 \mathrm{GJ} / \mathrm{t})$. 
Citation: Kumar A, Holuszko M, Janke T (2018) Separating Inorganics from the Non-metal Fraction of the Processed Waste PCBS Using Heavy Liquid Separation. Int J Waste Resour 8: 355. doi:10.4172/2252-5211.1000355

Page 5 of 9

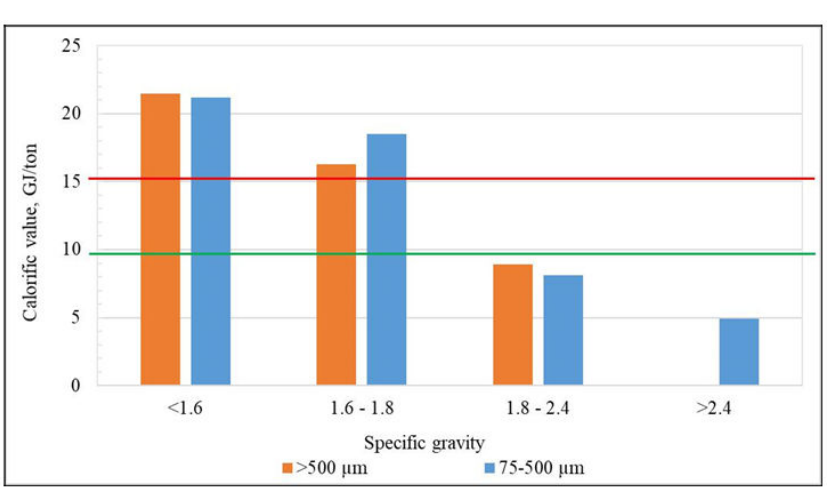

Figure 8: Calorific value of different SG fractions compared to feed for $>500 \mu \mathrm{m}$ and $75-500 \mu \mathrm{m}$ samples.

As expected, the results were consistent with the LOI results. The $<1.6$ and 1.8-2.4 SG products have similar calorific values, whereas 1.6-1.8 SG has higher calorific value for the fine fraction than the coarse sample likely due to entrainment of lighter materials in this fraction. The calorific value of the lower density fraction $(<1.6)$ is higher due to higher resin/organic matter content. The energy value of lower density fraction was determined to be approximately $16-20 \mathrm{GJ} / \mathrm{t}$. For comparison, the calorific value of lignite coal is $16 \mathrm{GJ} / \mathrm{t}$, peat coal is $13-20 \mathrm{GJ} / \mathrm{t}$, and dry wood is $14-17 \mathrm{GJ} / \mathrm{t}$ which suggests that the lowdensity products have a high potential application for energy recovery, depending on the analysis of released gases and the metal concentration in the combustion ash. It also suggests that the separating the fiberglass from the NMF would improve the calorific value of the final product.

The TGA analysis was performed to confirm the results from LOI and to measure the thermal stability of various product streams. The results for the TGA in the air are shown in Figure 9. The result showed a similar trend as for the LOI and calorific value results. The final residual masses of $<1.6$ and $1.8-2.4$ SG were almost similar $(12.8 \%$ compared to $12.6 \%$ and $64.3 \%$ compared to $65.4 \%$ ) showing that these products will have similar LOI and similar calorific values. The residual weight for +1.6-1.8 SG product for fine is lower than that for the coarse (24.0\% compared to $33.2 \%)$ suggesting the finer sample has larger mass loss compared to the coarse sample which in turns will provide higher LOI and thus higher calorific value. The degradation temperature in all cases is approximately $3000 \mathrm{C}$ where the mass of the sample starts to drop due to the thermal breakdown of the epoxy/phenolic resins associated with samples.
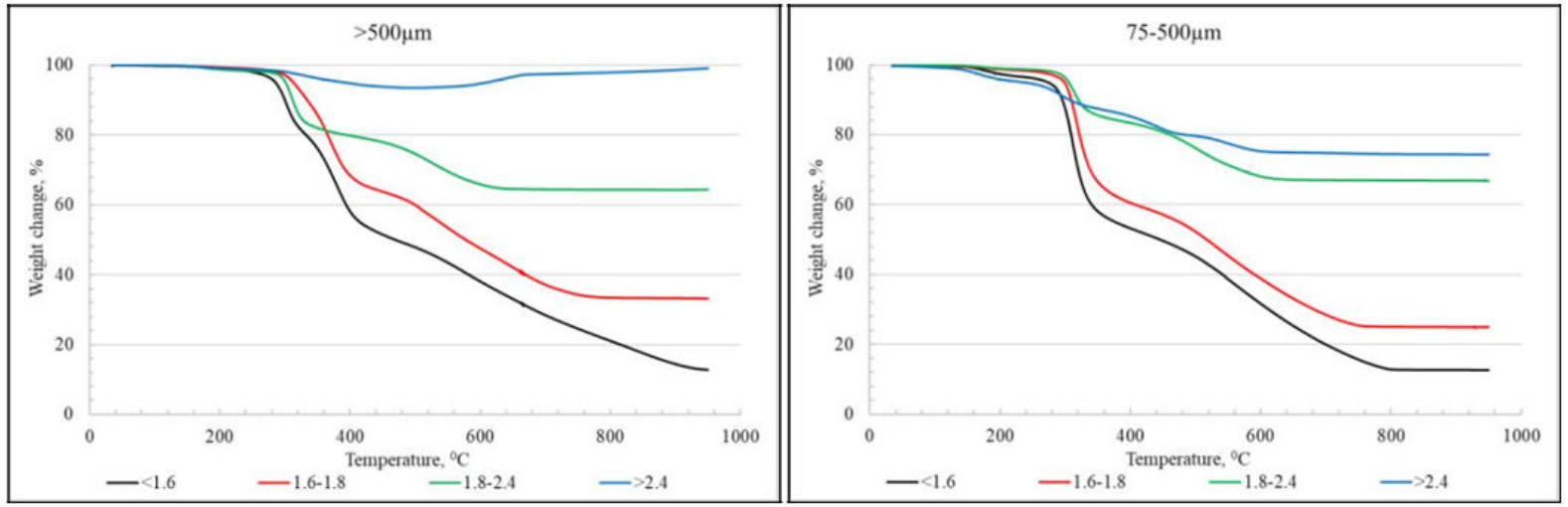

Figure 9: TGA in the air.

The TGA results for $>2.4$ SG product for coarse $(>500 \mu \mathrm{m})$ sample showed that the sample weight dropped until $\sim 7000^{\circ} \mathrm{C}$ and then started to rise again which suggest that the metals present in the system reacted to oxygen at this temperature forming oxides and thus increasing the weight. A TGA analysis of this sample in the inert (nitrogen) atmosphere is shown in Figure 10. The plot shows that the sample weight decreased to a minimum of $\sim 92 \%$ similar to the air atmosphere and then stabilized. In case of air, the weights decreased to $\sim 93 \%$ and then start increasing possibly due to the metal oxidation. 
Citation: Kumar A, Holuszko M, Janke T (2018) Separating Inorganics from the Non-metal Fraction of the Processed Waste PCBS Using Heavy Liquid Separation. Int J Waste Resour 8: 355. doi:10.4172/2252-5211.1000355

Page 6 of 9

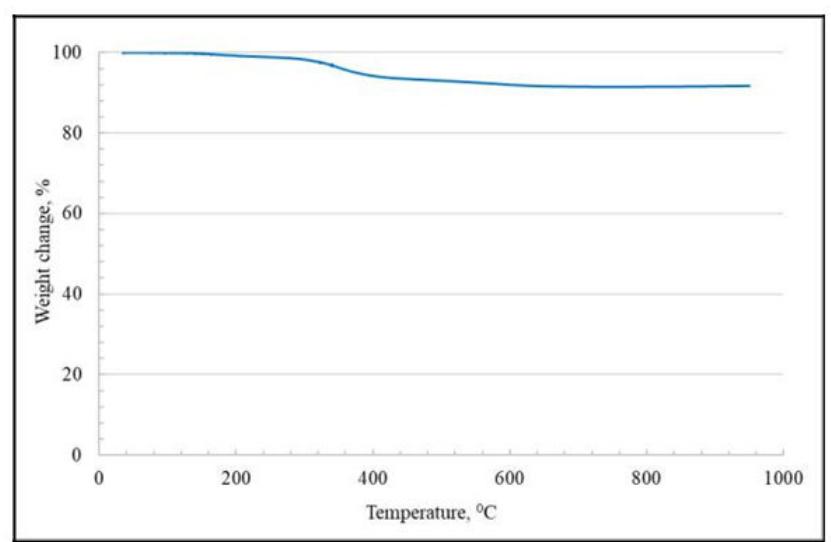

Figure 10: TGA test plots for $>2.4$ SG fractions for $>500 \mu \mathrm{m}$ sample in an inert atmosphere.

The feed grade for the samples used for this test is shown in Table 1. It shows that concentrations of most of the metals are negligible suggesting that most of the metals were already removed in the processing plant. The results for relatively higher-grade elements $(>500$ ppm or $0.05 \%$ ) are shown here (Table 1$)$.

\begin{tabular}{|c|c|}
\hline Element & Concentration (\%) \\
\hline Aluminum & 2.61 \\
\hline Antimony & 0.35 \\
\hline Calcium & 3.69 \\
\hline Copper & 0.78 \\
\hline Iron & 0.19 \\
\hline
\end{tabular}

Table 1: Results for relatively higher-grade elements

A metal content analysis of each specific gravity fraction from coarse and fine sizes was performed and the results for elements mentioned in Table 1 are shown in Figure 11. The metal content for almost all elements is highest in the heaviest specific gravity fraction (>2.4 SG). It shows that copper and iron content is very high in the coarse heavy fraction that confirms the negative LOI due to metal oxidation.
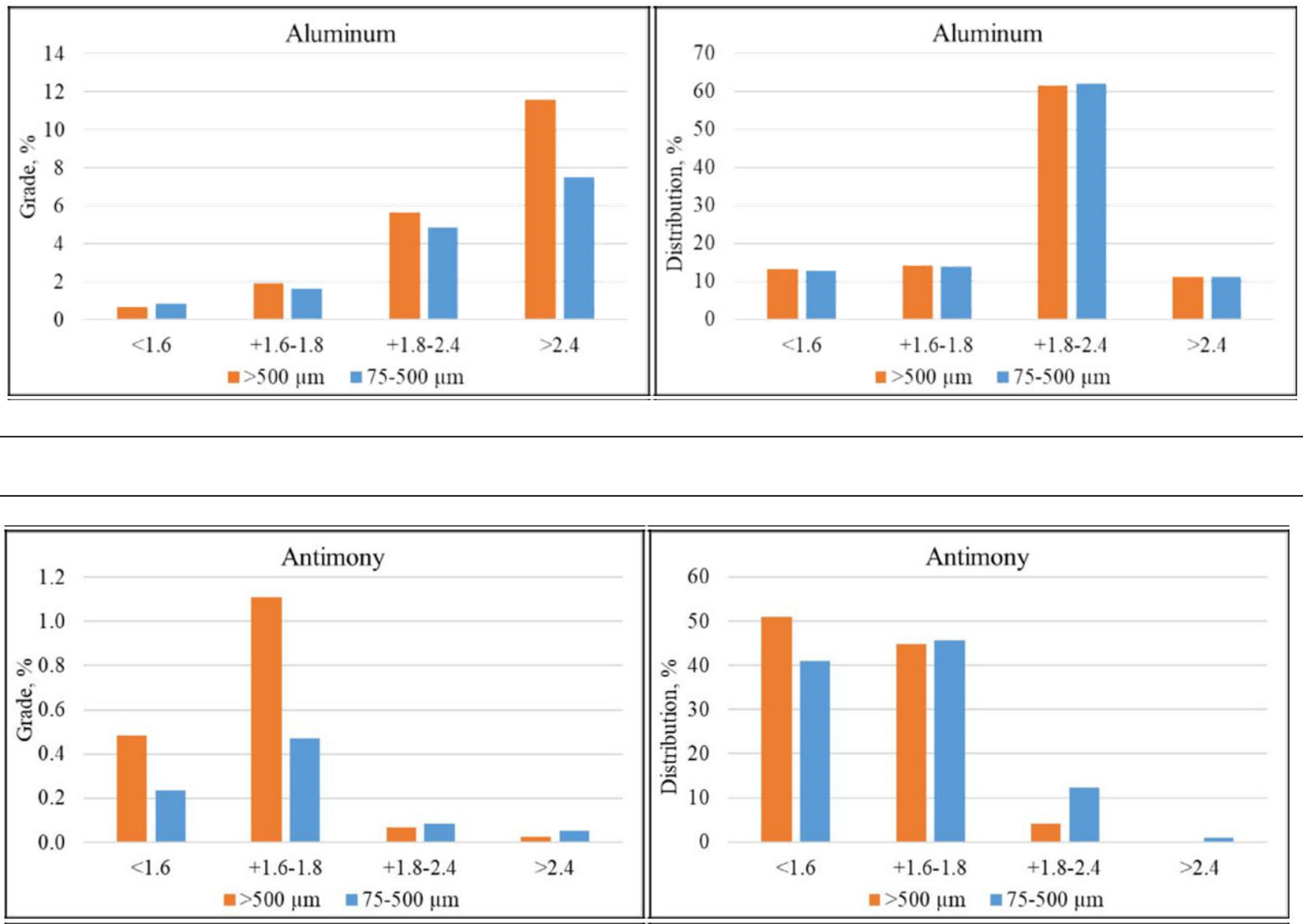
Citation: Kumar A, Holuszko M, Janke T (2018) Separating Inorganics from the Non-metal Fraction of the Processed Waste PCBS Using Heavy Liquid Separation. Int J Waste Resour 8: 355. doi:10.4172/2252-5211.1000355

Page 7 of 9
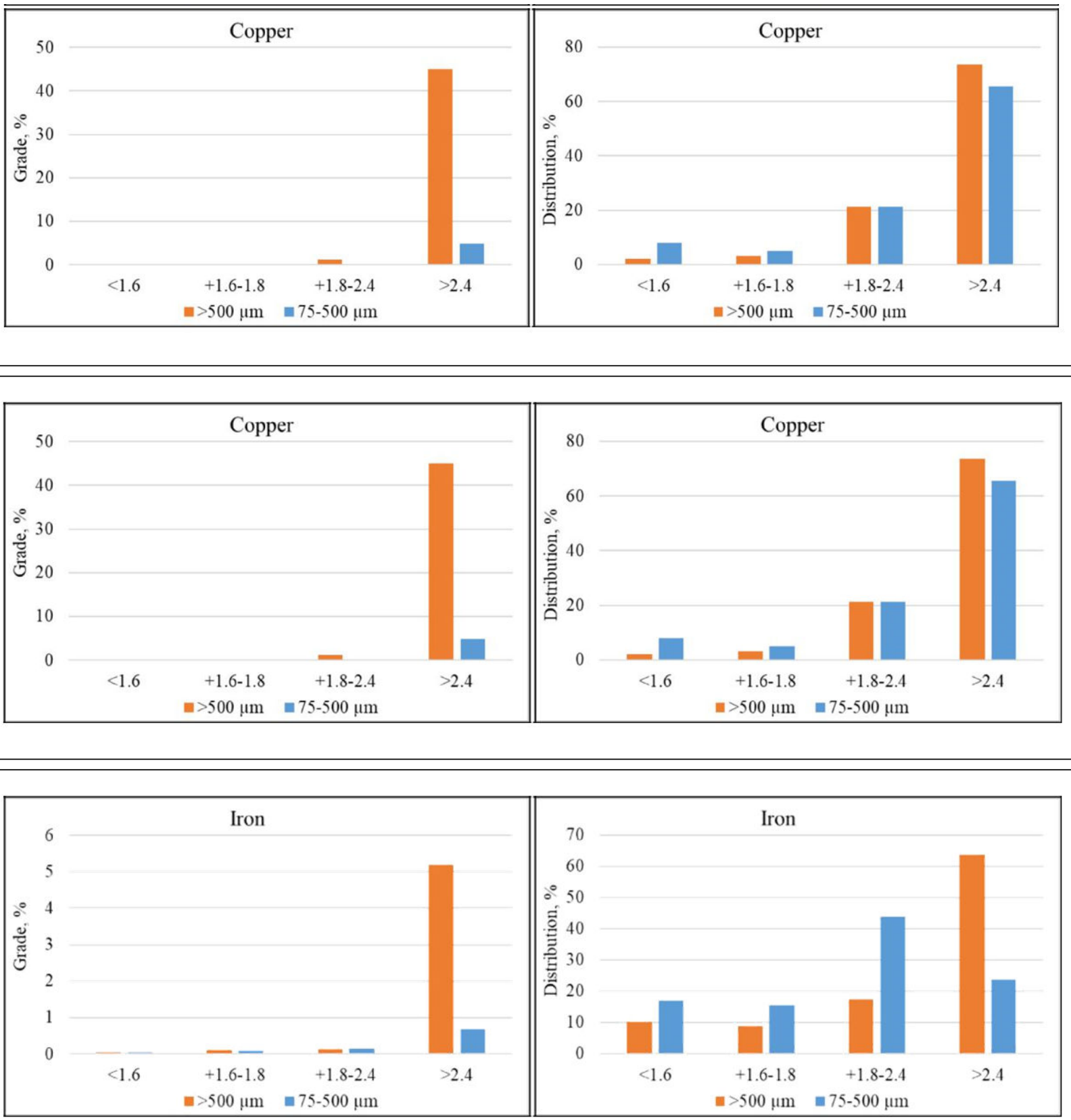

Figure 11: Metal content and distribution in different specific gravity fraction of $>500 \mu \mathrm{m}$ and $75-500 \mu \mathrm{m}$ sample.

Antimony is used in PCBs in the form of antimony oxide to improve the activity of flame retardants and is added to the PCB resins during the laminate production $[44,45]$. Since most of the resins reported to the $<1.6$ and 1.6-1.8 SG fraction, it should be expected that the antimony concentration and distribution are higher in this fraction. Calcium and aluminum are a part of the fiberglass matrix [14]. As such with the fiberglass reporting to the 1.8-2.4 SG fractions, it should be expected that the calcium and aluminum concentration and distribution in this density range should increase as well. Similar concentration of calcium and aluminum content in 1.8-2.4 SG and $>2.4$ SG fraction for fine sample suggests the similar nature of both stream that further confirms the similar LOI values for these two products (Figure 6, LOI $\sim 28 \%$ ). For copper and iron, the concentration is higher in the coarse heavier product than in the fine heavier product suggesting that long strands of metals which may not comminute during the shredding and size reduction processes and thus reported in the $>500 \mu \mathrm{m}$ size fraction.

Higher metal concentration in the heaviest fraction also shows that separating fiberglass from the NMF can be a potential solution for removing hazardous metals prior to incineration. 


\section{Conclusion}

The nonmetal fraction of the printed circuit boards contain a significant quantity of resins and fiberglass that was separated by using heavy organic liquids. A clear separation of organics from inorganics was obtained at a density of $1.8 \mathrm{~g} / \mathrm{cm}^{3}$. This separation could enhance the energy value and reduced the metal content of the concentrated organic fraction $(\mathrm{SG}<1.8)$. The lower specific gravity fraction $(<1.8)$ showed an energy content of approximately $21 \mathrm{GJ} / \mathrm{t}$, which is higher than the calorific value of lignite coal and dried wood and can likely be used for energy recovery. However, the byproducts of the energy recovery process/incineration, such as the flue gas and fly ash, should be evaluated for chemical and metal contamination. The heaviest density fraction showed an elevated metal content which can be used for further enhancing the overall metal recovery. The presence of $12 \%$, $45 \%$ and $5 \%$ aluminum, copper, and iron respectively were recorded in the products for the coarse sample $(>500 \mu \mathrm{m})$. The mid-density fraction, +1.8-2.4 SG, showed the presence of inorganic fiberglass that can be recycled as secondary raw materials depending on its physical and chemical properties which should be researched in more detail. The results showed that the dense media separation is an effective process to separate various fractions of the NMF present in PCBs. It will also reduce the amount of total waste to be disposed of and thus move towards the circular economy. Additional optical microscopy, scanning electron microscope, Fourier-transform infrared spectroscopy test and mechanical strength analysis will be needed to understand the nature of separated organic resins and inorganic fiberglass.

\section{Acknowledgment}

The author acknowledges Ronin8 Technologies Limited (Richmond, British Columbia) for their financial support as well as for providing samples, expert advice, and other necessary technical details. The author also acknowledges the Canadian funding agency MITACS Inc. for their financial assistance and ALS Environmental (Burnaby, British Columbia) for performing the external laboratory analysis.

\section{References}

1. Balde CP, Forti V, Gray V, Kuehr R, Stegmann P (2017) The Global Ewaste Monitor-2017. Bonn/Geneva/Vienna.

2. Cucchiella F, D’Adamo I, Lenny Koh SC, Rosa P (2015) Recycling of WEEEs: An economic assessment of present and future E-waste streams. Renew Sustain Energy Rev 51: 263-272.

3. Sthiannopkao S, Wong MH (2013) Handling e-waste in developed and developing countries: Initiatives, practices, and consequences. Sci Total Environ 463-464: 1147-1153.

4. Verma HR, Singh KK, Mankhand TR (2016) Dissolution and separation of brominated epoxy resin of waste printed circuit boards by using dimethyl formamide. J Clean Prod 139: 586-596.

5. Lin KH, Chiang HL (2014) Liquid oil and residual characteristics of printed circuit board recycle by pyrolysis. J Hazard Mater 271: 258-265.

6. Verma HR, Singh KK, Mankhand TR (2017) Liberation of metal clads of waste printed circuit boards by removal of halogenated epoxy resin substrate using dimethylacetamide. Waste Manag 60: 652-659.

7. Guo J, Guo J, Xu Z (2009) Recycling of non-metallic fractions from waste printed circuit boards: A review. J Hazard Mater 168: 567-590.

8. Marques AC, Cabrera Marrero JM, de Fraga Malfatti C (2013) A review of the recycling of non-metallic fractions of printed circuit boards. Springerplus 2: 521 .

9. Evangelopoulos P, Kantarelis E, Yang W (2015) Investigation of the thermal decomposition of printed circuit boards (PCBs) via thermogravimetric analysis (TGA) and analytical pyrolysis (Py-GC/MS). J Anal Appl Pyrolysis 115: 337-343.

10. Golev A, Schmeda-Lopez DR, Smart SK, Corder GD, McFarland EW (2016) Where next on e-waste in Australia? Waste Manag 58: 348-358.

11. Kumar A, Holuszko M, Espinosa DCR (2017) E-waste: An overview on generation, collection, legislation and recycling practices. Resour Conserv Recycl 122: 32-42.

12. Bizzo W, Figueiredo R, de Andrade V (2014) Characterization of Printed Circuit Boards for Metal and Energy Recovery after Milling and Mechanical Separation. Materials (Basel) 7: 4555-4566.

13. Luda MP (2011) Recycling of Printed Circuit Boards, in: Kumar, M.S (Ed.), Integrated Waste Management 2.

14. Szałatkiewicz J (2014) The Amount of PCBs in WEEE. Polish J Environ Stud 23: 2365-2369.

15. EPA (2012) Printed circuit board recycling methods.

16. Song Q, Wang Z, Li J (2012) Residents behaviors, attitudes, and willingness to pay for recycling E-waste in Macau. J Environ Manage 106: 8-16.

17. Das A, Vidyadhar A, Mehrotra SP (2009) A novel flowsheet for the recovery of metal values from waste printed circuitboards. Resour Conserv Recycl 53: 464-469.

18. Duan H, Hu J, Yuan W, Wang Y, Yu D, et al. (2016) Characterizing the environmental implications of the recycling of non-metallic fractions from waste printed circuit boards. J Clean Prod 137: 546-554.

19. Hadi P, Xu M, Lin CSK, Hui CW, McKay G (2015b) Waste printed circuit board recycling techniques and product utilization. J Hazard Mater 283: 234-243.

20. Kaya M (2016) Recovery of metals and nonmetals from electronic waste by physical and chemical recycling processes. Waste Manag 57: 64-90.

21. Namias J (2013) The future of electronic waste recycling in the United States: Obstacles and domestic solutions. Columbia University, New York, United States.

22. Ning C, Lin CSK, Hui DCW, McKay G (2017) Waste Printed Circuit Board (PCB) Recycling Techniques. Top Curr Chem 375: 43.

23. Veit HM, de Freitas Juchneski NC, Scherer J (2014) Use of gravity separation in metals concentration from printed circuit board scraps. Rem Rev Esc Minas 67: 73-79.

24. Bosecker K (1997) Bioleaching: metal solubilization by microorganisms. FEMS Microbiol. Rev 20: 591-604.

25. Khaliq A, Rhamdhani M, Brooks G, Masood S (2014) Metal Extraction Processes for Electronic Waste and Existing Industrial Routes: A Review and Australian Perspective. Resources 3: 152-179.

26. Tatariants M, Yousef S, Sidaraviciute R, Denafas G, Bendikiene R (2017) Characterization of waste printed circuit boards recycled using a dissolution approach and ultrasonic treatment at low temperatures. RSC Adv 7: 37729-37738.

27. Tatariants M, Yousef S, Denafas G, Tichonovas M, Bendikiene R (2018) Recovery of gold, other metallic and non-metallic components of full-size waste random access memory. J Clean Prod 172: 2811-2823.

28. Yousef S, Tatariants M, Makarevicius V, Lukosiute SI, Bendikiene R, et al. (2018) A strategy for synthesis of copper nanoparticles from recovered metal of waste printed circuit boards. J Clean Prod 185: 653-664.

29. Yousef S, Tatariants M, Bendikiene R, Denafas G (2017) Mechanical and thermal characterizations of non-metallic components recycled from waste printed circuit boards. J Clean Prod 167: 271-280.

30. Hadi P, Ning C, Ouyang W, Xu M, Lin CSK, et al. (2015a) Toward environmentally-benign utilization of nonmetallic fraction of waste printed circuit boards as modifier and precursor. Waste Manag 35: 236-246.

31. Kumar A, Holuszko ME, Janke T (2018) Characterization of the nonmetal fraction of the processed waste printed circuit boards. Waste Manag 75: 94-102.

32. Zheng Y, Shen Z, Ma S, Cai C, Zhao X, et al. (2009b) A novel approach to recycling of glass fibers from nonmetal materials of waste printed circuit boards. J Hazard Mater 170: 978-982. 
Citation: Kumar A, Holuszko M, Janke T (2018) Separating Inorganics from the Non-metal Fraction of the Processed Waste PCBS Using Heavy Liquid Separation. Int J Waste Resour 8: 355. doi:10.4172/2252-5211.1000355

Page 9 of 9

33. Sohaili J, Muniyandi SK, Mohamad SS (2012) A review on printed circuit boards waste recycling technologies and reusing of recovered nonmetallic materials. Int J Sci Eng Res 3: 138-144.

34. Zheng Y, Shen Z, Cai C, Ma S, Xing Y (2009a) The reuse of nonmetals recycled from waste printed circuit boards as reinforcing fillers in the polypropylene composites. J Hazard Mater 163: 600-606.

35. Li J, Gao B, Xu Z (2014) New Technology for Separating Resin Powder and Fiberglass Powder from Fiberglass-Resin Powder of Waste Printed Circuit Boards. Environ Sci Technol 48: 5171-5178.

36. Zhang G, Wang H, He Y, Yang X, Peng Z, et al. (2017b) Triboelectric separation technology for removing inorganics from non-metallic fraction of waste printed circuit boards: Influence of size fraction and process optimization. Waste Manag 60: 42-49.

37. Zhang G, He Y, Wang H, Zhang T, Yang X, et al. (2017a) Application of triboelectric separation to improve the usability of nonmetallic fractions of waste printed circuit boards: Removing inorganics. J Clean Prod 142: 1911-1917.

38. Engineeringtoolbox (2014) Densities of common solids.

39. NetComposites (2004) Resin Formul.

40. Tube Properties (2005) Properties of Carbon Fiber Tubes.

41. Kentucky Geological Survey (2017) Float-Sink (Washability) Test.

42. Burt RO, Mills C (1984) Gravity concentration technology. Elsevier.

43. Taggart AF (1945) Handbook of mineral dressing, 1st ed. John Wiley and Sons.

44. EPA (2015) Flame retardants in printed circuit boards.

45. Five Winds International (2001) Toxic and hazardous materials in electronics. 